\title{
Colonización y supervivencia de epibiontes sésiles en substratos artificiales similares a rizóforos de Rhizophora mangle (Rhizophoraceae) en La Mancha, México
}

\author{
Marcela Ruiz Guerrero ${ }^{1} \&$ Jorge López-Portillo ${ }^{2}$ \\ 1. Centro de Investigación en Alimentación y Desarrollo, A.C., Unidad Mazatlán en Acuicultura y Manejo Ambiental, \\ Mazatlán, Sinaloa, México; marcela@ciad.mx \\ 2. Red de Ecología Funcional, Instituto de Ecología, A.C., Xalapa, Veracruz, México; jorge.lopez.portillo@inecol.mx
}

Recibido 27-VI-2016. Corregido 25-I-2017. Aceptado 28-II-2017.

\begin{abstract}
Colonization and survival of sessile epibionts on artificial substrates similar to rhizophores of Rhizophora mangle (Rhizophoraceae) at La Mancha, Mexico. La Mancha coastal lagoon, in the Gulf of Mexico, has an inlet that opens and closes seasonally, causing important fluctuations in flood-levels and exposing the intertidal zone to high solar radiation, high temperatures and desiccation. With the aim to examine the accumulated effect of variation in flood-levels on colonization and survival of sessile intertidal invertebrates on artificial substrates in a fringe mangrove forest, a field experiment was conducted from November-2000 to September-2001. A total of 72 PVC-stakes $2 \mathrm{~m}$ long were encased with cement to resemble rhizophores (prop roots) of Rhizophora mangle. They were then embedded in mud at four sampling stations (18 per station: 9 in a shaded site and 9 in a sunny site), and groups of 6 were collected from each sampling station ( 3 from each shaded site and 3 from each sunny site) without replacement at the end of each climatic period (Eastern winds, dry and rainy). The open-closed condition of the inlet and the flood-level were monitored during different days throughout the 308-day experiment period. Temporal, spatial and vertical distribution of Species richness (S) and abundance of living and dead individuals were registered, and the data was analyzed by ANOVA. Only seven species colonized the substrates, the majority of them were recorded throughout the year and in all sampling stations. There was no seasonal succession of species. At the end of the experiment, only $20 \%$ of the epibionts had survived. The polychaete Ficopomatus miamiensis was the most abundant, but had significantly lower survival than the other species (0-14\%). Epibionts preferred to settle in shaded sites rather than sunny ones regardless of the sampling station and the climatic season. In conclusion, artificial substrate colonization was successful but the majority of epibionts died because of long exposure and desiccation after the inlet was opened by local fishermen. Manual digging of the sand-barrier by local fishermen, disrupts the natural flooding cycles of the lagoon, and could severely impact the future development of mangrove communities. Rev. Biol. Trop. 65 (2): 745-761. Epub 2017 June 01.
\end{abstract}

Key words: mangrove, coastal lagoon, ephemeral estuarine inlet, intertidal invertebrates, flood levels, desiccation, isolation, anthropic impact.

Las raíces aéreas, o rizóforos, del mangle rojo (Rhizophora mangle L.) proporcionan un substrato firme para el establecimiento de comunidades complejas y muy diversas de invertebrados y algas (Hogarth, 1999). Numerosos estudios, la mayoría realizados en costas del Mar Caribe, se han desarrollado para comprender los patrones de composición, distribución, abundancia y diversidad de estas comunidades de epibiontes, ya sea analizando a las raíces in situ o extrayéndolas del medio natural para procesarlas con mayor detenimiento en los laboratorios (Londoño-Mesa, Polanía, \& Vélez, 2002; Márquez \& Jiménez, 2002; García \& Palacio, 2008; Quiceno \& Palacio, 2008; Díaz \& Rützler, 2009; Wulff, 2009; Cedeño, Jiménez, Pereda, \& Allen, 2010; Quirós \& Arias, 2013; Acosta, Betancourt 
\& Prieto, 2014; Ruiz \& López-Portillo, 2014). Otra alternativa para el estudio de estas comunidades son los experimentos de campo en los cuales, a través del establecimiento de diferentes substratos artificiales que semejan las raíces del mangle rojo, se abordan temas de interés tales como la colonización y la sucesión por la epifauna. Los substratos más empleados han sido los azulejos y paneles de cerámica (Bingham, 1992; Marques-Silva et al., 2006), paneles de asbesto (Sutherland, 1980), paneles de madera (Marques-Silva et al., 2006), placas de acrílico (Bingham, 1992; Elliott, Persad, \& Webber, 2012), geles de agar (Hunting, Van der Geest, Krieg, Van Mierlo, \& Van Soest, 2010) y tubos y pilotes de PVC (Wulff, 2004; Díaz \& Liñero-Arana, 2006).

De manera general, los resultados de estos experimentos demuestran que los epibiontes tienen la facultad de colonizar el substrato duro que se les provee, y pueden, o no, desarrollar los mismos ensambles de especies que en las raíces en sus respectivos sistemas costeros. Adicionalmente, se ha observado que hay diferencias en las tasas de reclutamiento y en la longevidad de los individuos que logran establecerse, pero estas diferencias están más relacionadas con otros factores, como las condiciones ambientales y geomorfológicas de los sistemas costeros, la latitud y zona biogeográfica, las historias de vida de las especies y los efectos antrópicos (Sutherland, 1980; Bingham, 1992; Romero-Murillo \& Polanía, 2008; Ruiz \& López Portillo, 2014), que con el tipo de substrato utilizado en el experimento.

Uno de los trabajos clásicos con substratos artificiales fue el desarrollado por Sutherland (1980) en Bahía de Buche, Venezuela, quien determinó que para este sistema tropical, en el cual las condiciones ambientales son relativamente estables, la tasa de reclutamiento de la mayoría de las especies de epibiontes fue baja pero constante. El autor detectó también la ausencia de estacionalidad en el reclutamiento y que las especies parecen vivir por lo menos un año, resistiendo la invasión de nuevas larvas que buscan establecerse. Más tarde, Bingham (1992) encontró que, al menos para el Estado de la Florida, las condiciones ambientales más variables y estresantes actúan más a escalas espacio-temporales mayores sobre las abundancias de las comunidades epibentónicas, mientras que en el corto plazo y a pequeña escala, son los factores biológicos, como el suministro y longevidad de las larvas de las especies, quienes determinan la distribución y el desarrollo entre las poblaciones de adultos. Por otro lado, la sucesión de especies parece más marcada en islas oceánicas del Caribe comparada con sistemas costeros continentales, y se ha determinado que posiblemente este proceso depende de los ciclos climáticos (RomeroMurillo \& Polanía, 2008; Elliott et al., 2012).

El presente estudio describe los resultados de un experimento de colonización de substratos artificiales por epibiontes, según las condiciones ambientales tan particulares de la laguna costera de La Mancha, en México. Esta laguna tropical se comunica con el Golfo de México a través de una barrera arenosa que se cierra y abre estacionalmente (boca estuarina efímera), lo que ocasiona que los niveles de inundación de la laguna fluctúen a lo largo del año, de tal manera que la zona inundable se expone frecuentemente a la desecación y a altas temperaturas favorecidas por la radiación solar (Ruiz \& López Portillo, 2014). El experimento se realizó para conocer las especies de invertebrados sésiles que colonizan substratos artificiales en áreas de mangle rojo durante las diferentes épocas climáticas y para detectar si existen cambios estacionales en composición y abundancia de estos epibiontes, así como para conocer cuántos individuos sobreviven o mueren debido al efecto acumulado de la dinámica hidrológica de la boca estuarina.

\section{MATERIALES Y MÉTODOS}

Área de estudio: La laguna costera de La Mancha se ubica en el estado de Veracruz $\left(19^{\circ} 34^{\prime} \mathrm{N}-9^{\circ} 22^{\prime} \mathrm{W} \& 1^{\circ} 36^{\prime} \mathrm{N}-9^{\circ} 24^{\prime}\right.$ W, Fig. 1), en la región del Golfo de México caracterizada por tres épocas climáticas: 1) "nortes" (vientos otoño-invernales de finales de septiembre a marzo, pero son más intensos 


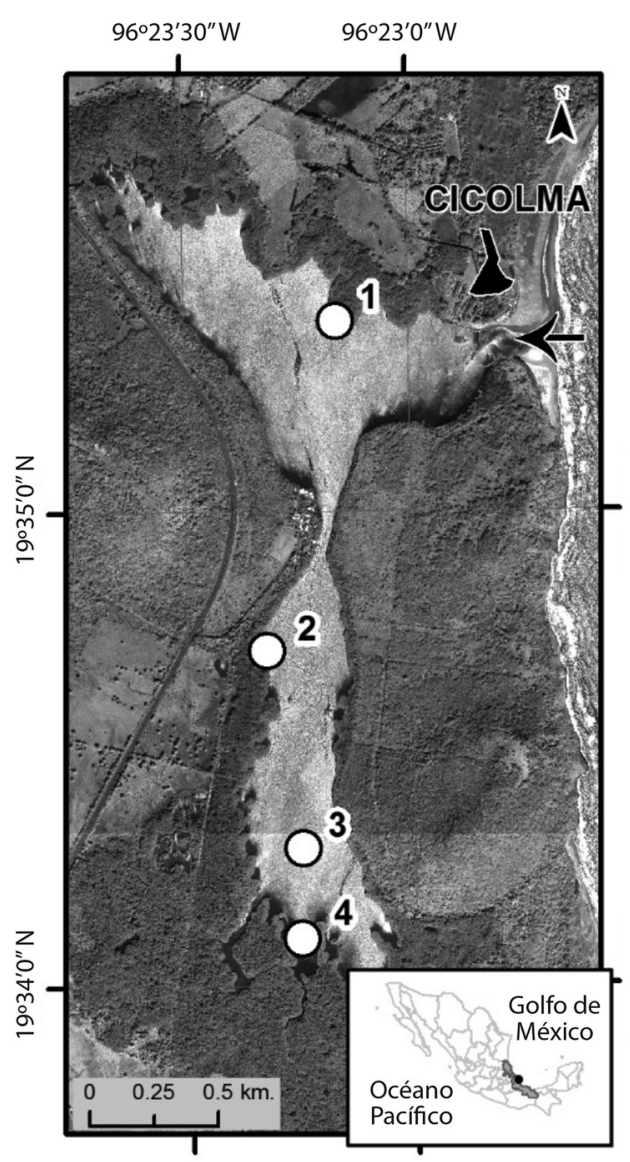

Fig. 1. Ubicación de cuatro estaciones de muestreo en el manglar de la laguna costera La Mancha, Veracruz, México. La flecha horizontal indica la boca de la laguna.

Fig. 1. Location of the four sampling stations at $\mathrm{La}$ Mancha coastal lagoon mangrove, Veracruz, Mexico. The horizontal arrow indicates the inlet of the lagoon.

de noviembre a febrero), 2) seca (de finales de febrero a mayo, pudiéndose extender hasta principios de junio) y 3) lluvias (de junio a septiembre) (Soto \& García, 1989; Ruiz, 2002). Esta laguna es relativamente pequeña, de unos $3 \mathrm{~km}$ de longitud norte-sur, tiene forma de reloj de arena, es muy somera en la parte norte $(0.20$ $\mathrm{cm}-1 \mathrm{~m}$ ) alcanzando una mayor profundidad en la parte sur (1-2.4 m) (Villalobos-Figueroa, De la Parra, Galván, Cacho, \& Izaguirre, 1984). El aporte de agua marina es a través de la boca estuarina, ubicada en la parte noreste, que se abre o se cierra dependiendo de la frecuencia e intensidad de los vientos, lluvias, mareas, y el arrastre litoral de sedimentos, mientras que el principal aporte de agua continental es a través de un arroyo, al sur de la laguna y por flujos subsuperficiales, al norte de la misma. Se ha registrado una diferencia promedio de $60 \mathrm{~cm}$ entre los niveles de inundación cuando la boca permanece varias semanas cerrada y acumula agua continental, en comparación cuando la boca se abre y libera toda el agua acumulada hacia el mar, quedando una capa somera de agua en el interior (Ruiz, 2002).

Estaciones de muestreo: Se establecieron cuatro estaciones de muestreo a lo largo del eje norte-sur de la laguna (Fig. 1): una estación cercana a la boca (E1), una estación cercana a la cintura de la laguna (E2), otra estación ubicada en un pequeño islote de mangle (E3), y la última estación localizada en la desembocadura del arroyo (E4), hacia el extremo sur de la laguna.

\section{Monitoreo hidrológico de la dinámica de} la boca estuarina y del nivel de inundación: Con la finalidad de conocer la periodicidad y tipo de eventos que provocan la apertura o cierre de la boca estuarina de la laguna de La Mancha, ya que estos inciden en el aumento o disminución del nivel de inundación en el interior de la laguna, se registró la condición (abierta o cerrada) de dicha barra arenosa en diferentes fechas al azar, procurando tener al menos un registro al mes, desde octubre de 2000 hasta septiembre de 2001. Además, en cada una de las estaciones de muestreo se midió el nivel de inundación, con un cordel graduado en centímetros sujetado a una plomada en su extremo distal, en la orilla del manglar, por triplicado, en siete fechas distribuidas durante el período del experimento. Los datos de los niveles de inundación se analizaron mediante un ANDEVA de dos vías para explorar diferencias entre épocas y estaciones de muestreo.

Diseño experimental: Se diseñaron substratos artificiales que pretendieron semejar raíces aéreas (rizóforos) de mangle rojo. Para ello 
se tomaron tubos de PVC de $4 \mathrm{~cm}$ de diámetro y $2 \mathrm{~m}$ de largo, a los cuales se les realizaron pequeñas perforaciones a todo lo largo de su superficie, y luego se les cosió un forro de malla mosquitera de plástico de $1 \mathrm{~mm}$ de luz de malla (Fig. 2A). Los tubos forrados se cubrieron con una preparación a base de cemento plástico marca CEMEX, arena, agua y colorante para cemento color café marca UNIBLOCK, para aparentar una superficie rugosa y oscura (Fig. 2B). Los tubos se colocaron en el substrato fangoso a unos $20 \mathrm{~cm}$ de profundidad y se ataron en su extremo superior a tallos o ramas de mangle rojo para una mejor sujeción (Fig. 2C). El 8 de noviembre 2000, se colocaron 18 tubos en la franja de manglar rojo en cada estación de muestreo (72 tubos en total). Se consideró pertinente incluir en el diseño
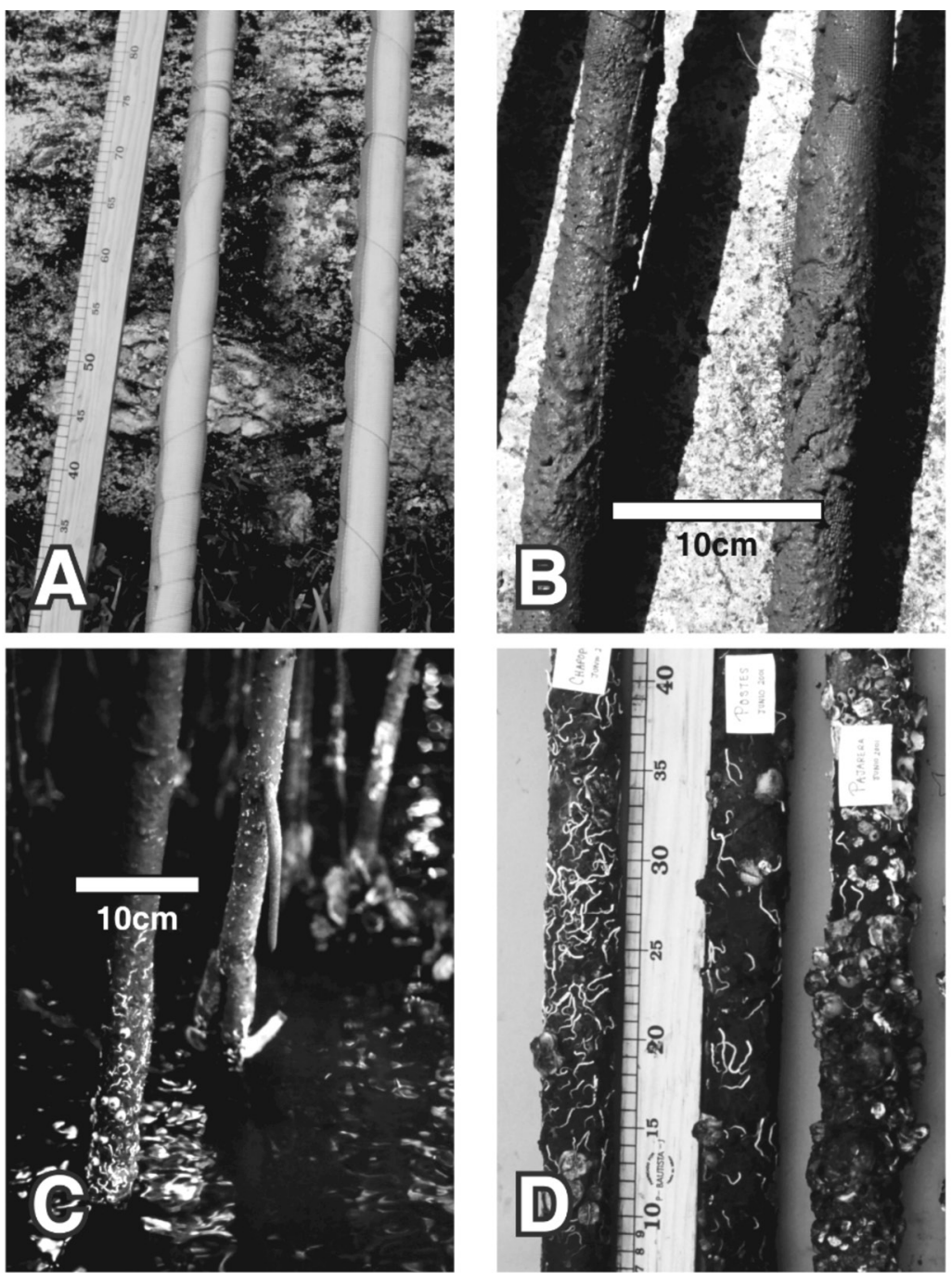

Fig. 2. Elaboración, colocación y recolecta de substratos artificiales: A) Tubos PVC forrados con malla mosquitera, B) Recubrimiento con cemento, C) Substrato artificial (al frente, a mano izquierda) y rizóforo de mangle rojo (atrás, a mano derecha), D) Substratos colonizados por epibiontes.

Fig. 2. Preparation, placement and collection of artificial substrates: A) PVC-stakes lined with nylon mesh, B) Cement coating, C) Artificial substrate (front, left-hand side) and red mangrove rhizophore (behind, right-hand side), D) Substrates colonized by epibionts. 
experimental el factor de insolación de los substratos, con el objetivo de detectar si la supervivencia de epibiontes se incrementa o disminuye dependiendo de la ubicación de los substratos en el microhábitat, por lo que de cada grupo de 18 tubos, 9 se instalaron en sitios sombreados (bajo el dosel de los árboles) y 9 en sitios soleados (fuera del dosel de los árboles). Tres tubos de cada sitio sombreado y 3 de cada sitio soleado se retiraron de cada estación de muestreo al término de cada época climática. El primer retiro se realizó en marzo 2001, a los 130 días de iniciado el experimento, a finales de la época de nortes. El segundo se realizó en el día 210 del experimento, en junio 2001, finalizada la época seca, y el tercero en el día 308, en septiembre 2001, a finales de la época de lluvias. Los tubos retirados fueron introducidos en bolsas de plástico, en seco, y llevados al Laboratorio del Centro de Investigaciones Costeras de La Mancha (CICOLMA; Fig. 1), donde se procesaron inmediatamente, aproximadamente una hora después de haber sido recolectados.

\section{Colonización y Supervivencia/Mortali-} dad de epibiontes en los substratos artificiales: En el laboratorio, cada tubo se analizó en intervalos de diez centímetros (nombrados horizontes sensu Ruiz \& López-Portillo, 2014), excluyendo la parte que estaba enterrada en el fango, y siguiendo un orden ascendente hacia la parte que estaba amarrada a los árboles de mangle (Fig. 2D). En cada horizonte (H), las especies de invertebrados sésiles se retiraron del substrato con un cuchillo a la vez que se iban revisando con ayuda de aguja y pinzas de disección, para distinguir y cuantificar los individuos catalogados como vivos (conchas, exoesqueletos y tubos calcáreos con presencia de organismos en su interior, considerándolos como "vivos" por presentar el organismo en estado fresco y húmedo, con coloración, con estructuras corporales definidas, a veces con movimiento pero no como requisito indispensable, sin necesidad de extraerlos completamente de sus tubos o exoesqueletos, únicamente verificando su presencia en el interior) y los individuos catalogados como muertos (conchas, exoesqueletos y tubos calcáreos vacíos o con materia orgánica seca, amorfa o en descomposición). Las especies móviles también se retiraron de los substratos, se cuantificaron y se identificaron pero no fueron tenidas en cuenta para el presente estudio. En cada horizonte de cada tubo se determinó la Riqueza específica (S = número de especies) y la abundancia (número de individuos vivos y muertos) de cada una de las especies sésiles que colonizaron los substratos. Si la especie tenía hábito colonial, cada colonia se contó como un individuo. Con base en los individuos cuantificados en el total de los tubos se determinó el porcentaje de individuos vivos (\% supervivencia) y de individuos muertos (\% mortalidad) para cada especie en cada época climática.

Para la mayor certeza, en la determinación taxonómica de los epibiontes, se solicitó la ayuda de especialistas del ICMyL-UNAM. Los especímenes se conservaron en etanol al $70 \%$ y se depositaron para su resguardo en el Laboratorio de Ecología Funcional en el INECOL, en Xalapa, México.

Variación espacio-temporal de la Riqueza específica y la abundancia: La Riqueza específica y la abundancia fueron las variables de respuesta que separadamente se analizaron con ANDEVA de tres vías, considerando cada tubo como una réplica (Ruiz \& López-Portillo, 2014; Zar, 1996), para explorar diferencias entre los siguientes factores: 1) épocas climáticas, estaciones de muestreo e individuos vivos/ muertos, y 2) estaciones de muestreo, horizontes e individuos vivos/muertos.

Análisis del efecto de la insolación: Se realizó un ANDEVA de tres vías para explorar diferencias de la supervivencia (individuos vivos y muertos) entre tubos de sitios sombreados y de sitios soleados, épocas y estaciones de muestreo, con la Riqueza específica y la abundancia como variables de respuesta. Además, se realizó una prueba a posteriori de Duncan (Sokal \& Rohlf, 1995) para comparar entre tratamientos. 


\section{RESULTADOS}

Monitoreo hidrológico de la dinámica de la boca estuarina y del nivel de inundación: La boca estuarina se cerró y abrió varias veces durante la época de nortes, pero permaneció abierta durante toda la época de lluvias (Fig. 3A). No se pudo registrar el número máximo de días en que la boca estuarina puede permanecer cerrada en condiciones naturales, y por ende, el nivel de inundación máximo que se puede alcanzar en el interior de la laguna, debido a la frecuente intervención de los pescadores locales, quienes llevan a cabo ciertas maniobras para asegurar que entren peces a la laguna y así tener producto para vender, entre otras razones. Cuando el nivel de la laguna era elevado, los pescadores excavaban con pala manual un canal angosto sobre la barrera arenosa, adelantando así el proceso natural de vertido hacia el mar del agua contenida en la laguna, lo que después de unas pocas horas resulta en un nivel bajo de inundación. Se logró registrar un mínimo de veinte días

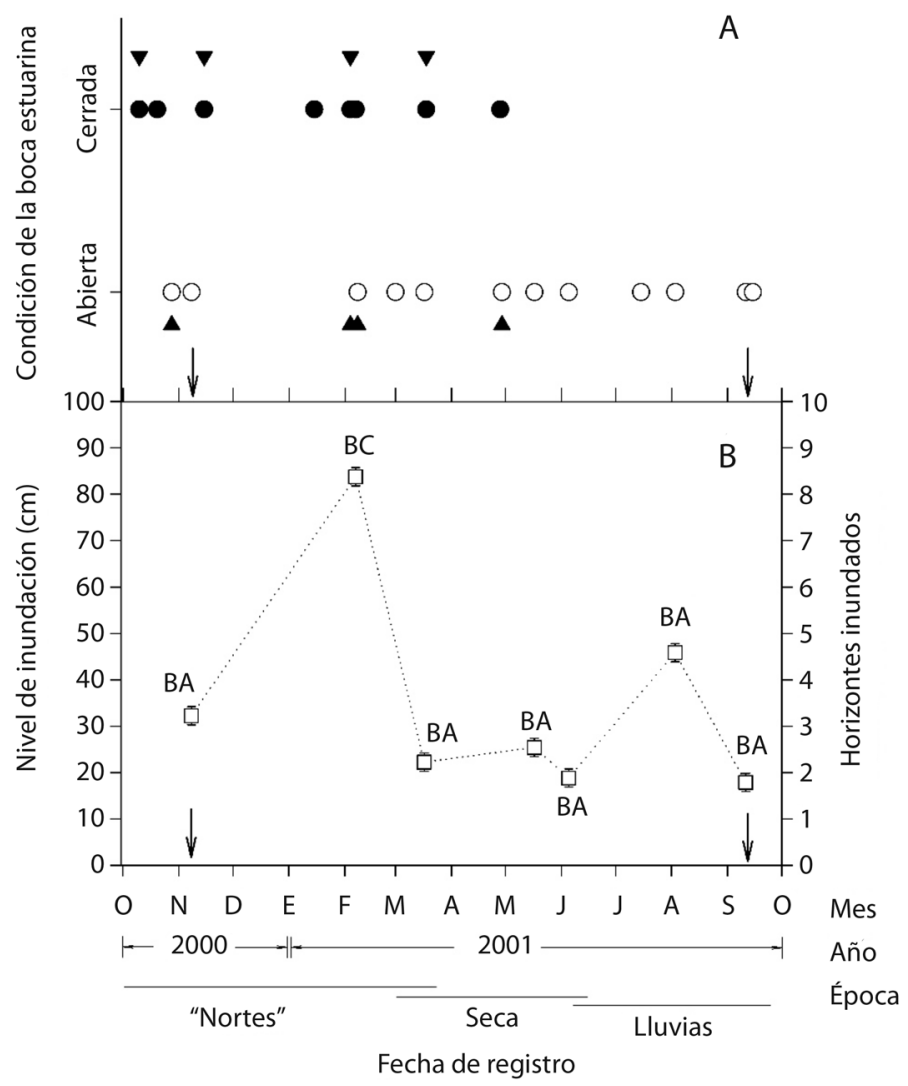

Fig. 3. Monitoreo hidrológico de la laguna La Mancha, de octubre-2000 a septiembre-2001, durante tres épocas climáticas. A) Condición abierta $(\circ)$ y cerrada $(\bullet)$ de la boca estuarina y los principales eventos causantes de su apertura $(\boldsymbol{\Delta}=$ Acción de los pescadores) o de su cierre ( $\boldsymbol{\nabla}=$ Vientos Nortes $), B)$ Nivel de inundación ( \pm ES) y horizontes experimentales inundados en siete fechas y en ambas condiciones de la boca $(\mathrm{BA}=$ Boca abierta, $\mathrm{BC}=$ Boca cerrada). Las flechas verticales indican el inicio y término del experimento.

Fig. 3. Hydrological monitoring of La Mancha lagoon, from October-2000 to September-2001, along three climatic seasons. A) Open ( $\circ$ ) and closed $(\bullet)$ inlet condition at La Mancha lagoon and major events that cause inlet to open $(\boldsymbol{\Delta}=$ Manual digging by fishermen) or close ( $\boldsymbol{\nabla}=$ Nortes winds), B) Flood levels $( \pm$ SE) and flooded experimental horizons at seven different days and in both inlet condition $(\mathrm{BA}=$ Open inlet, $\mathrm{BC}=$ Closed inlet). Vertical arrows indicate the start and end dates of the experiment. 
y un máximo de cuarenta días en que los pescadores permitieron que la boca estuarina permaneciera cerrada durante la época de nortes. Por ejemplo, el último viento del norte de la temporada 2000-2001 se registró la noche del 17 de marzo, lo que ocasionó que la barrera arenosa se cerrara, y cuando ya llevaba 40 días cerrada, los pescadores la reabrieron el 29 de abril (Fig. 3A).

El mayor nivel de inundación de la laguna de La Mancha se registró en época de nortes en febrero $(83.7 \mathrm{~cm} \pm 1.9$ Media \pm Error estándar, entre el horizonte $\mathrm{H} 8$ y H9), coincidiendo con la boca estuarina cerrada (Fig. 3B). Los más bajos niveles se observaron cuando la boca estuarina estaba abierta en la época seca en junio (18.8 $\mathrm{cm} \pm 1.9)$, y al final de la época de lluvias en septiembre $(17.8 \mathrm{~cm} \pm 1.9$; horizontes $\mathrm{H} 1$ y H2 inundados; Fig. 3B). La diferencia entre extremos de inundación fue de $66 \pm 1.9$ $\mathrm{cm}$. Se registró un importante incremento en el nivel de inundación, que alcanzó hasta el H5, durante eventos de lluvia constante que duró dos días en el mes de agosto. Respecto a la variación espacial, se registró un gradiente de inundación que se incrementó de E1 $(25.1 \mathrm{~cm}$ \pm 1.5 ; horizontes 1-3 inundados) hacia E4 (46.1 $\mathrm{cm} \pm 1.5 ; \mathrm{H} 1$ al H5 inundados). El análisis de varianza mostró diferencias significativas entre fechas $\left(\mathrm{F}_{6,56}: 143, \mathrm{p}<0.0001\right)$, entre estaciones de muestreo $\left(\mathrm{F}_{3,56}: 35.7, \mathrm{p}<0.00001\right)$, y en la interacción $\left(\mathrm{F}_{18,56}: 19.7, \mathrm{p}<0.0001\right)$.

Colonización de epibiontes: El experimento duró 308 días. De los 72 tubos instalados, 69 (96\%) fueron colonizados por al menos una especie y 3 (4\%) no presentaron ningún rastro de epibionte sésil. Siete especies de invertebrados sésiles que colonizaron los substratos artificiales fueron: el briozoo Membranipora sp., el poliqueto Ficopomatus miamiensis (Treadwell, 1934), cuatro moluscos bivalvos Crassostrea rhizophorae (Guilding, 1828), Ischadium recurvum (Rafinesque, 1820), Isognomon alatus (Gmelin, 1791) y Mytilopsis leucophaeata (Conrad, 1831); y un crustáceo balánido Amphibalanus eburneus (Gould, 1841) (Cuadro 1).
Se contabilizó un total de 24843 individuos, de los cuales 4276 (17\%) se registraron como "vivos" y 20567 (83\%) como "muertos". La colonización total en términos de Riqueza específica fue igual para las tres épocas climáticas $(S=6)$, mientras que en términos de abundancia total fue similar entre las épocas de nortes y seca (8 183 y 8178 individuos, respectivamente) pero mayor para la época de lluvias (8 482 individuos) (Cuadro 1). La especie con el mayor número de individuos reclutados fue $F$. miamiensis (19 019) y la del menor número fue I. alatus (1). Cinco de las siete especies estuvieron presentes en las tres épocas climáticas, y de las dos restantes, I. ala$t u s$, se registró sólo en la época seca mientras que Membranipora sp., estuvo ausente en esa misma época (Cuadro 1).

La colonización de epibiontes por estaciones de muestreo fue mayor en la $\mathrm{E} 4$, con 10 315 individuos de seis especies, 3376 (33\%) vivos y 6939 (67\%) muertos. La abundancia acumulada en esta estación representó a su vez el $41 \%$ de la abundancia total anual de epibiontes registrados en este experimento en La Mancha. Todas las especies colonizaron las cuatro estaciones de muestreo (Fig. 4), excepto I. alatus, del que solamente se registró un individuo en E2 (por lo que se omite el gráfico de la especie). Sin embargo, Membranipora sp. presentó mayores abundancias en E1, I. recurvus y $C$. rhizophorae en E3, y $F$. miamiensis, M. leucophaeata y A. eburneus en E4 (Fig. 4).

En cuanto a la colonización vertical (Fig. 5), se registró el máximo de nueve horizontes con presencia de epibiontes, es decir, las especies pudieron establecerse sobre los tubos hasta una altura máxima entre 80 y $90 \mathrm{~cm}$ desde el fondo fangoso. Las especies que se establecieron en los nueve horizontes, al menos en una de las épocas climáticas, fueron $F$. miamiensis y M. leucophaeata, mientras que I. alatus tuvo más afinidad por el horizonte más cercano al fondo (H1). El único horizonte colonizado por las siete especies de epibiontes fue H1.

Supervivencia/Mortalidad: De manera general, los porcentajes de mortalidad 


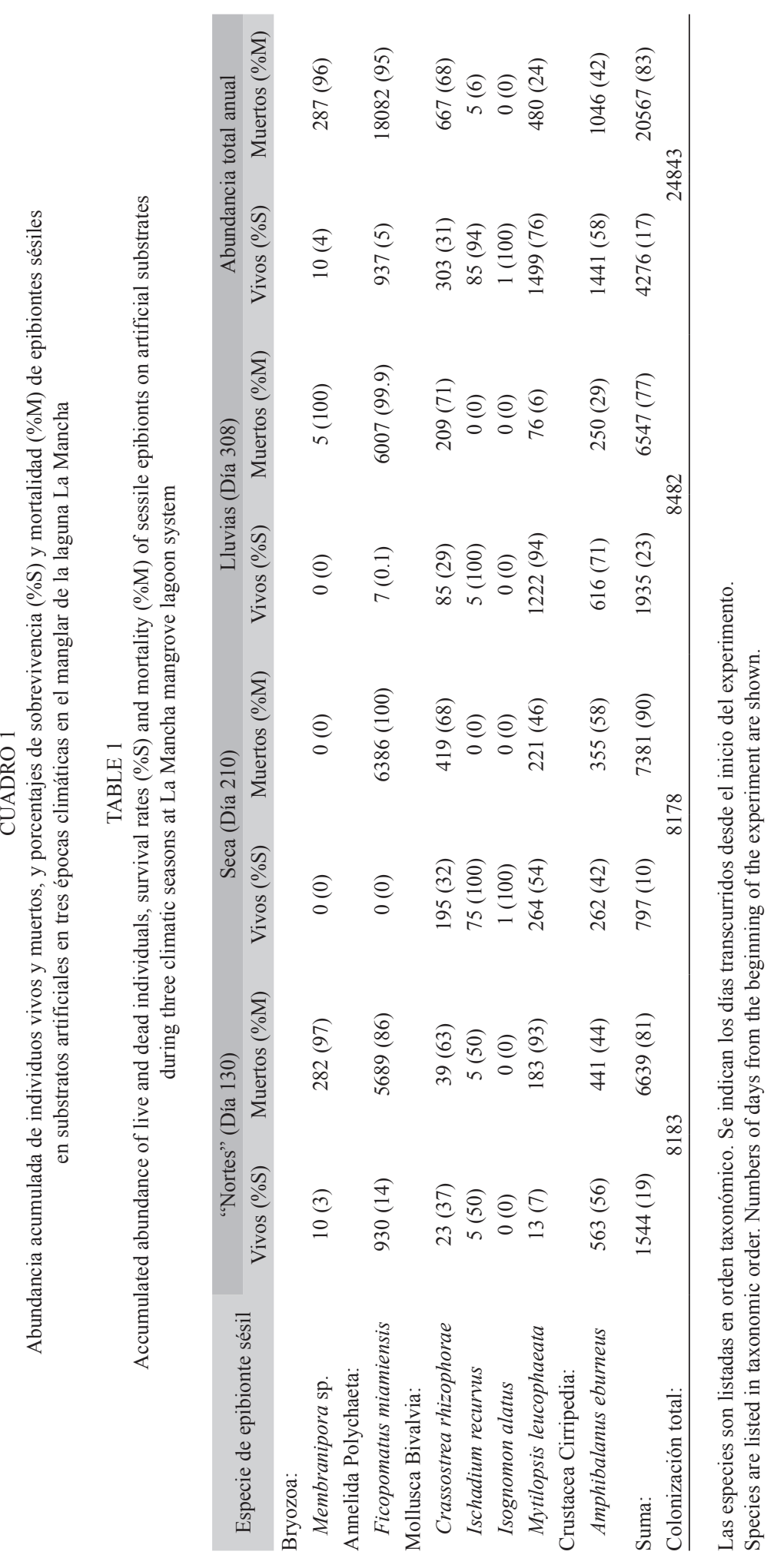




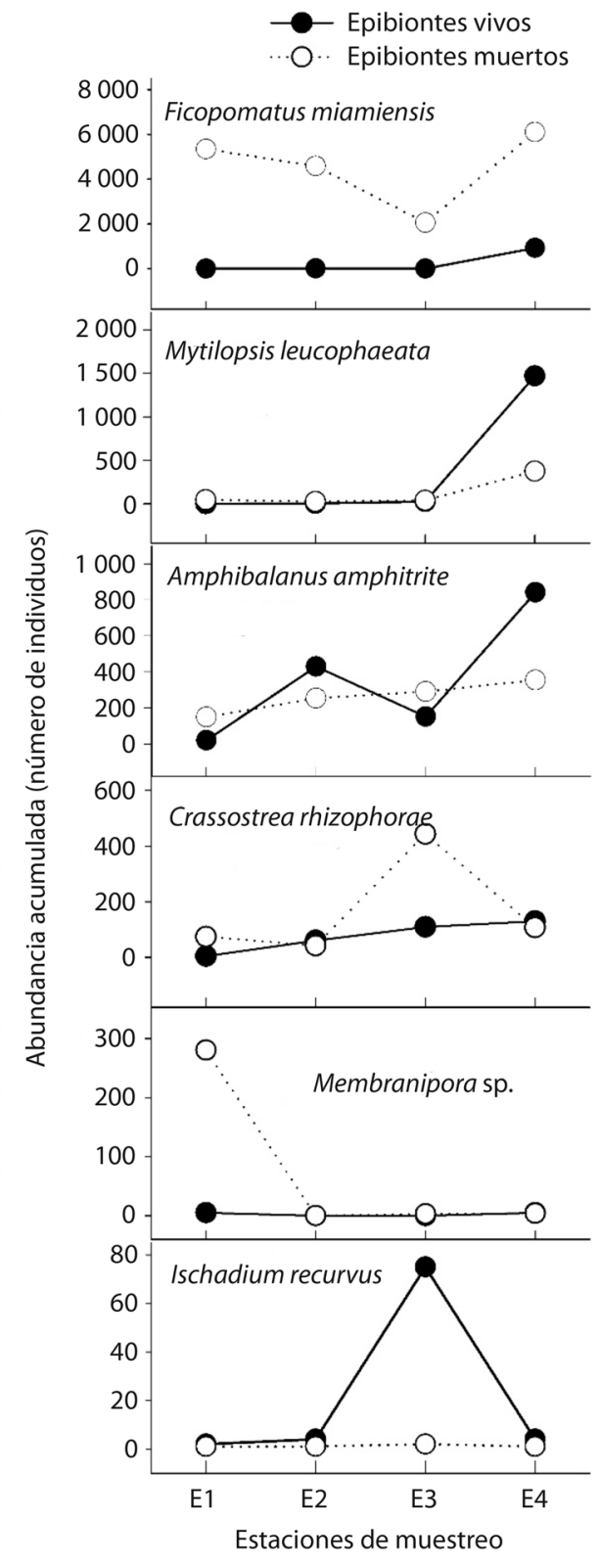

Fig. 4. Abundancia acumulada (número de individuos vivos y muertos) por especie de epibionte sésil en substratos artificiales en cuatro estaciones de muestreo en la laguna de La Mancha, México. Las especies aparecen en orden de importancia numérica.

Fig. 4. Accumulated abundance (live and dead individuals) per species of sessile epibiont on artificial substrates at four sampling stations in La Mancha lagoon, Mexico. Species are listed in numerical order of importance. de los epibiontes fueron mayores que los porcentajes de supervivencia en las tres épocas climáticas, aunque en lluvias (77\%) no fue tan alto como en nortes $(81 \%)$ ni tan extremo como en la época seca (90 \%) (Cuadro 1). El poliqueto $F$. miamiensis presentó los mayores porcentajes de mortalidad particularmente en la época seca $(100 \%)$ y en la época de lluvias (99.9\%). Por el contrario, el falso mejillón M. leucophaeata fue la especie con el mayor número de individuos vivos (1 499), y en lluvias presentó su más alta supervivencia (94\%). El ostión C. rhizophorae aumentó su abundancia en la época seca, pero en las tres épocas climáticas, presentó mayor mortalidad que supervivencia. De las tres especies con las menores abundancias en todo el experimento, dos de ellas, I. alatus e I. recurvus, registraron altas supervivencias, mientras que en la tercera, Membranipora sp., altas mortalidades. El balano A. eburneus presentó menos variación en la relación supervivencia/mortalidad en sus poblaciones respecto a las otras especies, obteniendo en el recuento total al final del experimento, un número ligeramente mayor de sobrevivientes que de muertos (Cuadro 1).

La mayoría de las especies sobrevivieron más en la E4 que en el resto de las estaciones, a excepción de $I$. recurvus que sobrevivió más (97\%) en la E3 (Fig. 4). El poliqueto $F$. miamiensis fue la especie que de manera más constante presentó más individuos muertos que vivos en toda la laguna, con mortalidades de $100 \%$ y $99 \%$ en el norte y centro de la laguna, hasta $87 \%$ en el sur en la E4. Aunque Membranipora sp. colonizó preferentemente la E1, murieron el $98 \%$ de sus individuos (Fig. 4).

En cuanto a la supervivencia vertical, todas las especies tuvieron sus mayores porcentajes de supervivencia (68 - $98 \%$ ) en $\mathrm{H} 1$ y H2 (Fig. 5), y una alta mortalidad (100\%) en los horizontes más altos. Por otro lado, la única especie que de manera constante presentó mayor número de individuos muertos que vivos en los nueve horizontes fue $F$. miamiensis. 

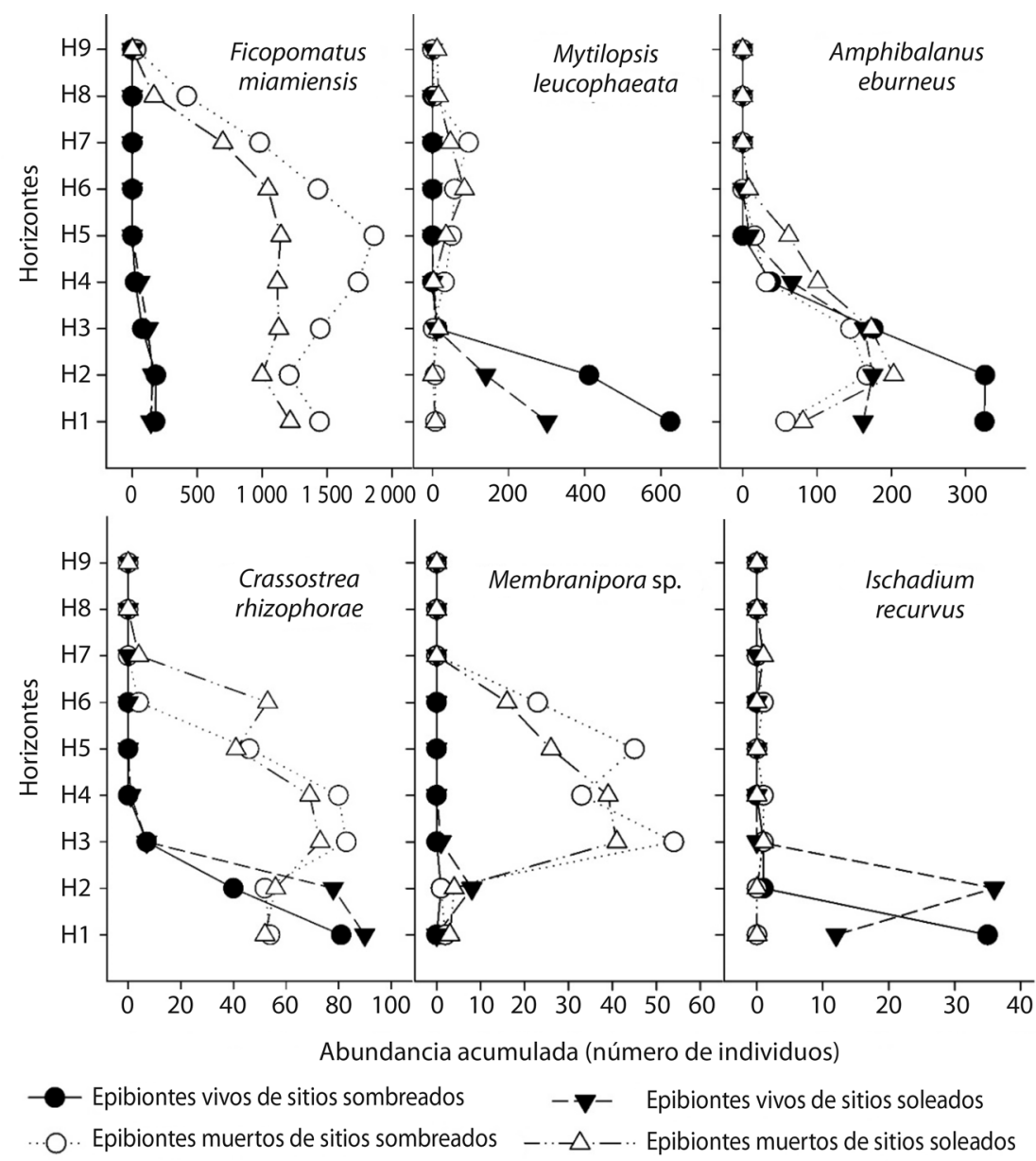

Fig. 5. Distribución vertical (por horizonte) de la abundancia acumulada (individuos vivos y muertos) por especie de epibionte sésil en substratos artificiales, distinguiendo entre sitios sombreados y sitios soleados, en la laguna de La Mancha, México. Las especies aparecen en orden de importancia numérica.

Fig. 5. Vertical distribution (by horizon) of accumulated abundance (live and dead individuals) per species of sessile epibiont on artificial substrates, differentiated between shaded and sunny sites at La Mancha lagoon, Mexico. Species are listed in numerical order of importance.

Variación espacio-temporal de la Riqueza específica y la abundancia: Los resultados de los ANDEVA fueron más contundentes para los datos de abundancia que para los de Riqueza específica, por lo tanto se describen a continuación los resultados más relevantes de los primeros. La abundancia de epibiontes vivos se mantuvo significativamente por debajo de la abundancia de epibiontes muertos en las tres épocas climáticas y en las cuatro estaciones de muestreo (Fig. 6; $\mathrm{F}_{6,1272}: 3.45, \mathrm{p}=0.002$ ).
La abundancia de epibiontes vivos aumentó de E1 (norte de la laguna) hacia E4 (al sur), mientras que la abundancia de los epibiontes muertos decreció hacia E3 en la época seca y en la de lluvias (Fig. 6). Por otro lado, se encontraron diferencias significativas entre la abundancia de epibiontes vivos versus muertos y su distribución vertical por horizontes en las cuatro estaciones de muestreo (Fig. 7; $\mathrm{F}_{24,1224}$ : $12.919, \mathrm{p}=0.00001)$. Los epibiontes vivos, con abundancia baja, solamente se registraron 


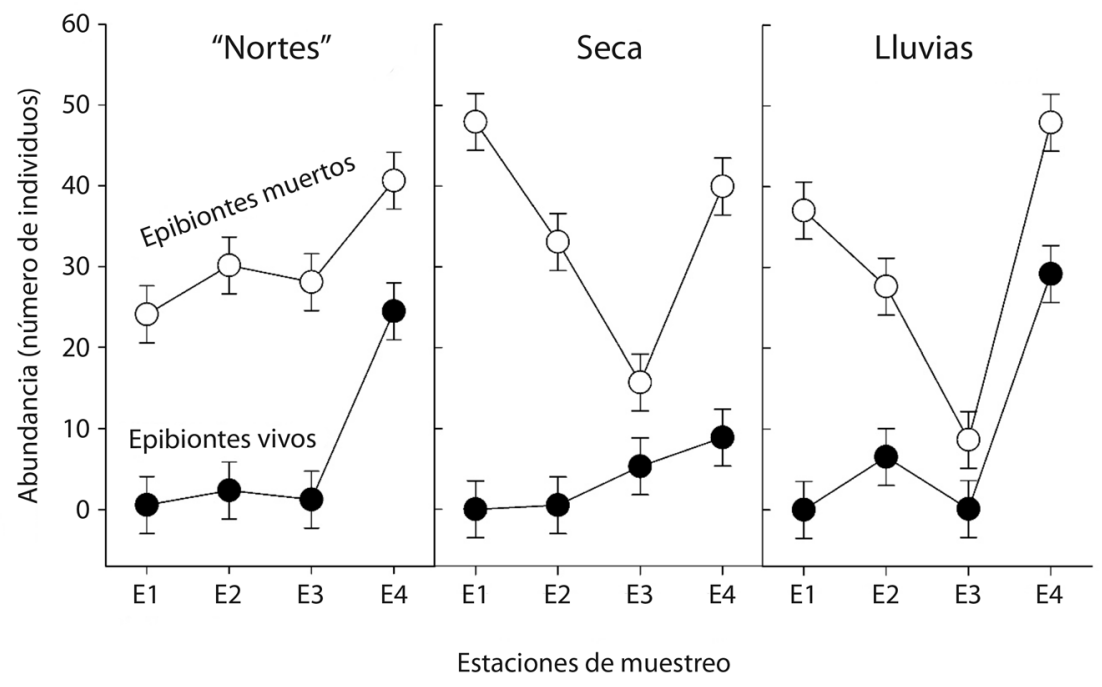

Fig. 6. Abundancia (Media \pm ES) de epibiontes sésiles vivos y muertos en substratos artificiales en tres épocas climáticas y cuatro estaciones de muestreo en la laguna de La Mancha, México.

Fig. 6. Live and dead sessile epibionts abundance $(M e a n \pm S E)$ on artificial substrates at three climatic seasons and four sampling stations at La Mancha lagoon, Mexico.

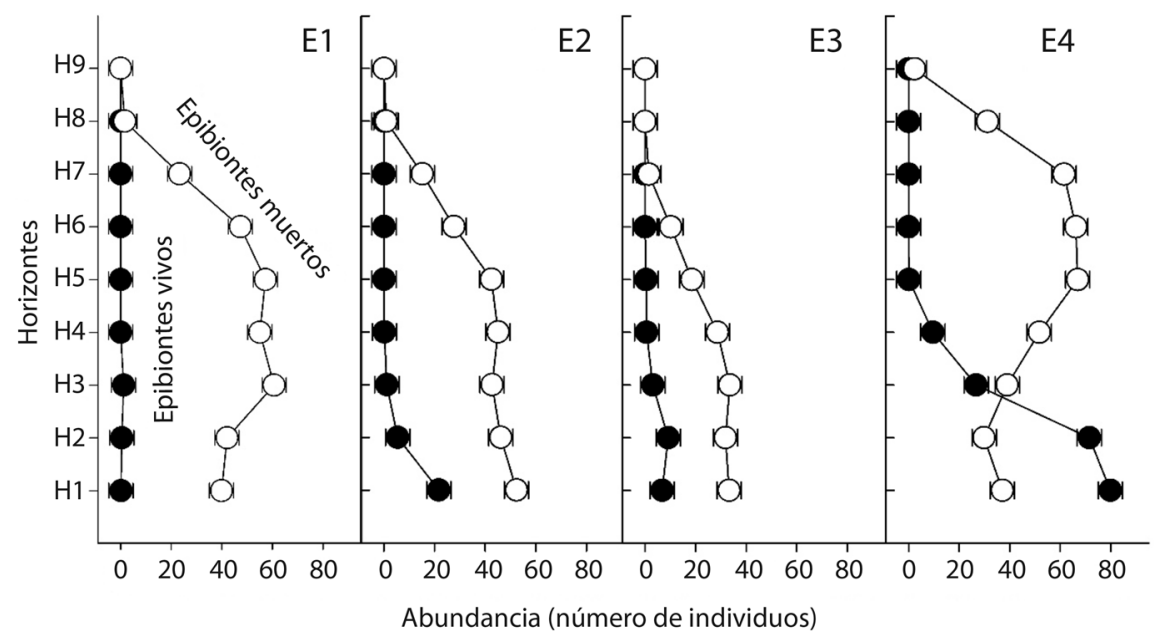

Fig. 7. Abundancia (Media \pm ES) de epibiontes sésiles vivos y muertos en substratos artificiales en horizontes experimentales y cuatro estaciones de muestreo en la laguna de La Mancha, México.

Fig. 7. Live and dead sessile epibionts abundance (Mean \pm SE) on artificial substrates at experimental horizons and four sampling stations at La Mancha lagoon, Mexico.

en los horizontes más cercanos al fondo (H1H5), y los epibiontes muertos, con una marcada mayor abundancia, se registraron en un mayor número de horizontes, sobre todo en la E4 alcanzaron hasta el H9. Se determinó que en las estaciones E1, E2 y E3 los epibiontes muertos abundaron en los horizontes $\mathrm{H} 1 \mathrm{al} \mathrm{H6}$, y la estación E4 fueron más abundantes en los horizontes $\mathrm{H} 4$ a H7. Cabe resaltar la E4, en la cual, hubo mayor abundancia de epibiontes vivos que de muertos en $\mathrm{H} 1$ y $\mathrm{H} 2$, y a partir del $\mathrm{H} 3$ al H9 el comportamiento fue inverso, o sea, abundaron más los epibiontes muertos que los vivos (Fig. 7). 


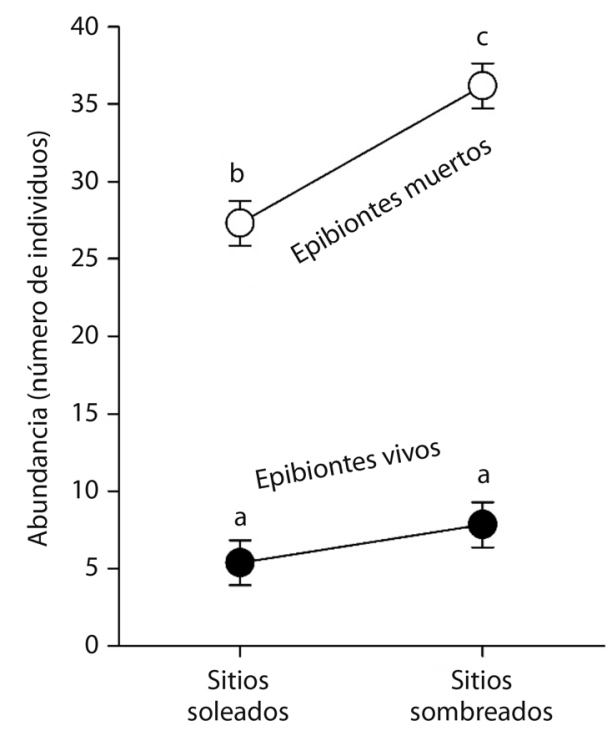

Fig. 8. Comparación de la abundancia (Media \pm ES) de epibiontes sésiles vivos y muertos en substratos artificiales entre sitios soleados y sitios sombreados en la laguna de La Mancha, México. Las letras minúsculas representan grupos similares.

Fig. 8. Comparison between the abundance (Mean \pm SE) of live and dead sessile epibionts on artificial substrates in shaded and sunny sites at La Mancha lagoon, Mexico. Lower case letters represent similar groups.

Análisis del efecto de la insolación: No se encontró un efecto significativo de la insolación para los datos de Riqueza específica de los epibiontes sésiles que colonizaron los tubos $\left(\mathrm{F}_{1,1280}: 0.63, \mathrm{p}=0.42\right)$ pero sí para los datos de abundancia, encontrándose que hubo mayor abundancia de epibiontes muertos en los sitios sombreados que en los sitios soleados (Fig. 8; $\left.\mathrm{F}_{1,1280}: 4.89, \mathrm{p}=0.02\right)$. No se encontraron diferencias significativas entre épocas climáticas ( $\mathrm{p}$ $=0.402)$ ni entre estaciones de muestreo $(\mathrm{p}=$ 0.61 ) en la abundancia de epibiontes en sitios soleados versus sitios sombreados. La mayor abundancia de epibiontes muertos en los sitios sombreados respecto a los sitios soleados fue más evidente para las especies Membranipora sp., F. miamiensis y C. rhizophorae en los horizontes H3 al H7 (Fig. 5).

\section{DISCUSIÓN}

En el transcurso del presente experimento se observó que los vientos nortes fueron los principales causantes del cierre de la boca estuarina de La Mancha, y las maniobras de excavación de los pescadores locales, las causantes de su apertura, por encima de los eventos de apertura natural. A pesar de la intervención de los pescadores en la dinámica de la boca estuarina, se confirma que: 1) el nivel de inundación aumenta cuando la boca estuarina está cerrada, lo que puede tomar varios días o varias semanas; 2) el nivel también aumenta cuando hay grandes aportes de agua por lluvias aun estando abierta la boca estuarina, y su duración es de pocas horas o días; y 3) el nivel de inundación decrece drásticamente en pocas horas, con la apertura de la barrera arenosa. Este comportamiento de la boca estuarina efímera, concuerda con los resultados de Lara-Domínguez, Day, Yáñez-Arancibia y Sáinz-Hernández (2006), quienes encontraron que La Mancha es un fuerte exportador neto de agua al océano, principalmente en nortes y en lluvias. Además, se pudo observar que la boca estuarina se mantuvo predominantemente abierta durante el año, por lo que los niveles de inundación de la laguna son relativamente bajos, mismo comportamiento observado por Psuty et al. (2009).

Los resultados del presente experimento reflejan el importante efecto de la fluctuación del nivel de inundación sobre la colonización y baja supervivencia de los epibiontes sésiles. Los epibiontes colonizaron eficientemente los tubos en todos los horizontes inundados a lo largo del año, lo cual indica que, de manera general, hubo un suministro de larvas en la columna de agua que estaban dispuestas a establecerse en los substratos en cuanto hubo disponibilidad de espacio vertical, y las larvas que se establecieron pertenecieron a las especies que en ese momento se estaban reproduciendo. Sin embargo, la mortalidad de los epibiontes 
establecidos fue total en los horizontes intermedios y altos de los tubos, debido a la repentina exposición a la desecación cada vez que bajaba el nivel de inundación. Por el contrario, la supervivencia fue mayor en los escasos dos o tres horizontes, que permanecieron inundados a lo largo del año, tal como lo registraron Ruiz y López-Portillo (2014) para los epibiontes en raíces de mangle rojo en esta misma laguna. La variabilidad en el nivel de inundación en la laguna de La Mancha no sólo impacta el desarrollo de los epibiontes sésiles de la zona intermareal del manglar, sino que se ha determinado que el impacto de dicha variabilidad también se manifiesta en el desarrollo del manglar y usos ecosistémicos. Se ha podido registrar que cuando los períodos de inundación son cortos y poco frecuentes, la comunidad arbórea de manglar que rodea a la laguna es mono específica y de baja altura, pero cuando los períodos de inundación son más largos y con mayor frecuencia, el manglar es más diverso y alcanza mayor altura (Hernández-Trejo, Priego-Santander, López-Portillo, \& Insunza-Vera, 2006). Esto indica lo favorable que resulta para el sistema, que se mantenga cerrada la barrera estuarina por un período relativamente largo.

De acuerdo con los resultados, la especie con el mayor porcentaje de mortalidad causada por el efecto acumulado de las fluctuaciones en los niveles de inundación, fue el poliqueto $F$. miamiensis. Esta especie fue capaz de colonizar nueve horizontes, pero, aunque es una especie intermareal con una resistencia comprobada de sobrevivir más de 24 horas de exposición a la desecación en granjas camaronícolas cuando se hacen los recambios de agua (Tóvar-Hernández \& Yáñez-Rivera, 2012), no pudo sobrevivir en los tubos colocados en La Mancha después de abrirse la boca estuarina, pues el período de desecación supera el tiempo de tolerancia de ésta, y de las demás especies intermareales, ya que puede tomar varios días o semanas para que se vuelvan a cubrir con agua. Sin embargo, la historia de vida de este poliqueto tubícola le permite seguir reproduciéndose y colonizando los substratos duros en La Mancha, a pesar de la baja profundidad prolongada de la laguna y de las pocas oportunidades que tiene de establecerse cuando hay más disponibilidad de espacio vertical durante la época de nortes. Tovar-Hernández y Yáñez-Rivera (2012) han determinado que las larvas planctotróficas de esta especie se establecen en los substratos duros a los 13 días de haber sido desovadas y tardan dos días en construir sus tubos y engrosarlos, además, se reproducen desde tallas muy pequeñas, desde $3.5 \mathrm{~mm}$ de largo. Por lo tanto, al menos para esta especie, en esos períodos de escasos 20-40 días que aproximadamente los pescadores dejaron cerrada la laguna de $\mathrm{La}$ Mancha, le fueron suficientes para colonizar, reproducirse, y persistir en este sistema, aunque es importante señalar que no alcanzó a formar, ni en los tubos, ni en las raíces de mangle, las colonias voluminosas en forma de arrecifes que se han registrado para especies del género Ficopomatus (Schwindt, De Francesco, \& Iribarne, 2004; Tovar-Hernández \& Yáñez-Rivera 2012), sino que apenas le dio tiempo de que se incrustaran individuos solitarios, o a formar asociaciones masivas pero rudimentarias.

No se observó una sucesión de las especies sésiles conforme transcurrieron las diferentes épocas climáticas, sino que la mayoría de ellas estuvieron presentes desde la primera etapa del experimento, y permanecieron desarrollándose en el año, de ahí que las diferencias en los datos de riqueza específica no fueran tan evidentes como con los datos de abundancia. Una de las especies que estuvo presente en todos los muestreos fue $C$. rhizophorae, que se ha determinado que se reproduce continuamente durante todo el año en las costas de Brasil (Lenz \& Boehs, 2011), y tal parece que también en La Mancha se reproduce con periodicidad, y probablemente sus individuos sean relativamente longevos. Los resultados parecen indicar que el aumento en la abundancia de ciertas especies en alguna de las épocas climáticas (particularmente nortes y lluvias), pudo deberse más a sus épocas reproductivas que a la duración de la exposición de los substratos, o a un proceso de sucesión de especies. Por ejemplo, en el caso de F. miamiensis, aparentemente la gran mayoría de sus individuos se establecieron 
desde la primera etapa del experimento, en nortes, cuando se alcanzaron los mayores niveles de inundación y cuando esta especie aprovechó el espacio vertical para establecerse, de ahí que se registraran tan altas mortalidades y cero, o escasos individuos vivos, en la segunda y tercera etapas del experimento. Igualmente para el caso de M. leucophaeata, que fue la única especie en la cual se observó un incremento en su abundancia hacia el día 308 del experimento, posiblemente tal incremento se debió más a que su temporada reproductiva, que es de junio a octubre (Rajagopal, Van der Gaag, Van der Velde, \& Jenner, 2005), coincidió hacia la etapa final de experimento (septiembre) que a un proceso de sucesión. La sucesión de especies que se ha observado en otros sistemas costeros sí tiene una relación con el tiempo de exposición a los substratos artificiales, por ejemplo en Jamaica, Elliott, et al. (2012) determinaron que los balanos e hidroides inician la colonización de placas de acrílico pero rápidamente son reemplazados por ascidias y briozoos. Sin embargo, cabe señalar que los resultados aquí presentados corresponden a un diseño experimental enfocado únicamente en las especies sésiles y para evaluar el impacto acumulado en ellas, además, son una primera aproximación y marcan la pauta para futuras investigaciones, especialmente sería interesante conocer la colonización y la sucesión de especies en etapas todavía más tempranas de las estudiadas aquí, pues se ha visto que en otro estudio realizado en el Caribe colombiano, a los 30 días de iniciado el experimento hubo claros indicios de colonización (Romero-Murillo \& Polanía, 2008).

Bingham (1992) comprobó que, al menos para el sistema costero estudiado en Florida, no siempre las condiciones ambientales más estresantes dan como resultado más bajas abundancias y diversidad de epibiontes, sino que más bien los patrones de distribución son una combinación de factores físicos y biológicos. Pero, para el caso de La Mancha, las condiciones ambientales estresantes, ocasionadas por la dinámica hidrológica de la boca estuarina, sí juegan un papel determinante en las bajas abundancias y altas mortalidades de los epibiontes sésiles de la zona intermareal del manglar, lo cual concuerda más con el modelo clásico para zonas intermareales rocosas, en el que las partes altas de un perfil de playa, o en este caso las partes altas de los tubos o substratos artificiales, son modeladas por factores físicos, y las partes bajas, que aquí serían los horizontes permanentemente inundados, por factores biológicos (Little \& Kitching, 1996).

El efecto de la insolación sobre la supervivencia de epibiontes ha sido escasamente estudiado en áreas de manglar, pero se sabe de la importancia que tiene en general la iluminación, aunada a la temperatura, y en ocasiones relacionada también con la profundidad, en el establecimiento y desarrollo de especies intermareales y submareales, y que muchas larvas siendo fotopositivas en el plancton se convierten en fotonegativas en el momento de colonizar un fondo marino (Reyes \& Campos, 1992; Little \& Kitching, 1996). En La Mancha, el análisis del efecto de la insolación sobre los epibiontes sésiles nos permitió observar que sí hubo preferencia por el microhábitat sombreado, con una mayor colonización pero con consecuente mayor mortalidad que en los substratos de sitios soleados, y por otro lado, los individuos que sobrevivieron al descenso del nivel de inundación no reflejaron diferencias significativas ni en riqueza ni en abundancia entre microhábitats, por lo que se puede apreciar que los horizontes permanentemente inundados tienen condiciones ambientales relativamente más estables. La preferencia de los epibiontes por colonizar substratos en sitios sombreados también fue observada en la Bahía Chengue, Colombia por Reyes y Campos (1992). En Belice se determinó experimentalmente que las esponjas Haliclona y Lisodendoryx, habitantes submareales y del intermareal bajo, pueden sobrevivir y logran regenerarse en individuos saludables después de exponerse seis horas al sol, pero Scopalina, que se distribuye verticalmente por debajo de las anteriores, solamente sobrevive dos horas cuando se expone a la desecación sin importar si es a la sombra o al sol (Rützler, 1995). Estos resultados permiten 
observar el diferente grado de resistencia de las especies a la desecación y a la insolación, pero en el caso de los epibiontes de La Mancha, el tiempo de exposición a estos factores es tan prolongado, que causa mortalidades del 100 $\%$ aun cuando las especies posean conchas y exoesqueletos que las protegen contra la pérdida de agua, incluso si son las más resistentes por habitar en la zona intermareal alta.

En conclusión, la dinámica hidrológica de la laguna de La Mancha dio como resultado el establecimiento en los substratos artificiales de únicamente siete especies de invertebrados intermareales, que en general permanecen en la laguna sin una clara sucesión de especies relacionada con las épocas climáticas. La mortalidad de los individuos fue significativamente mayor que la supervivencia, particularmente en las partes altas de los substratos, debido a los prolongados períodos de desecación cuando disminuye el nivel de inundación tras la apertura de la boca estuarina. La baja proporción de individuos sobrevivientes se mantuvo en un espacio vertical reducido a $20-30 \mathrm{~cm}$. Aparentemente, hay una importante sincronización de las historias de vida de los epibiontes sésiles con la alta variabilidad ambiental de La Mancha, y las especies colonizan preferentemente los substratos en sitios puntuales sombreados que en sitios puntuales soleados sin distinción de la época climática ni de la estación de muestreo en la laguna. Las maniobras de excavación de la barrera arenosa por los pescadores locales, que interrumpen el llenado máximo posible de la laguna y que se adelantan a la apertura natural de la boca estuarina, podrían traer consecuencias futuras en el desarrollo de las comunidades intermareales que habitan en la laguna de La Mancha.

\section{AGRADECIMIENTOS}

El presente trabajo es parte de la tesis de Maestría de M. Ruiz en el Posgrado del INECOL, quien agradece al CIAD-Mazatlán y a A.M. van der Heiden por la licencia laboral para la realización del presente estudio. Se agradece a J. Tolome, E. López-Barradas,
A. Martínez-Chacón, H. Barradas, F. Aguilar y A. García su apoyo en campo y a R. Monroy la elaboración de la Fig. 1. A los especialistas del ICMyL-UNAM: A. Granados Barba (Polychaeta), M. Reguero Reza (Mollusca) y J. Salgado (Crustacea) por la identificación taxonómica del material colectado. También se agradece a A. Castillo-Guerrero y a los revisores anónimos sus comentarios para mejorar este manuscrito y V. Williams su apoyo en la traducción al idioma inglés. Estudio financiado por el Proyecto CONACYT 25935-N.

\section{RESUMEN}

La laguna costera de La Mancha, en el Golfo de México, tiene una boca que se cierra y abre estacionalmente, lo que ocasiona importantes fluctuaciones en los niveles de inundación y que la zona intermareal se exponga frecuentemente a una alta radiación solar, altas temperaturas y su consecuente desecación. Se llevó a cabo un experimento de campo de Noviembre-2000 a Septiembre-2001 para evaluar el efecto acumulado de la variación en el nivel de inundación sobre la colonización y supervivencia de los invertebrados intermareales sésiles sobre substratos artificiales en el bosque de manglar de borde. Un total de 72 tubos de PVC de $2 \mathrm{~m}$ de largo fueron recubiertos con cemento para semejar rizóforos (raíces aéreas) de Rhizophora mangle. Luego fueron encajados en el fango en cuatro estaciones de muestreo (18 por estación: 9 en sitio sombreado y 9 en sitio soleado), y fueron retirados en grupos de 6 de cada estación de muestreo ( 3 de cada sitio sombreado y 3 de cada sitio soleado), sin reemplazo, al término de cada época climática (nortes, seca y lluvias). Además, se monitoreó la condición abierta-cerrada de la boca estuarina y el nivel de inundación durante el período de 308 días que duró el experimento. Se registró la distribución temporal, espacial y vertical de la Riqueza específica (S) y de la abundancia de individuos vivos y muertos, y los datos fueron analizados mediante ANDEVA. Únicamente siete especies colonizaron los substratos, la mayoría de ellas se registraron todo el año y en todas las estaciones de muestreo. No se observó una sucesión estacional de las especies. Al final del experimento, se obtuvo una supervivencia del $20 \%$ de los epibiontes. El poliqueto Ficopomatus miamiensis fue la especie más abundante pero presentó una supervivencia significativamente menor que las otras especies encontradas $(0-14 \%)$. Los epibiontes prefirieron establecerse en sitios sombreados que en sitios soleados sin importar la estación de muestreo ni la época climática. En conclusión, la colonización de los substratos artificiales fue exitosa pero la mayoría de los individuos murieron debido a la prolongada exposición y desecación después de que la boca estuarina fue abierta por los pescadores locales. 
Las maniobras de excavación de la barrera arenosa por los pescadores locales interrumpen los ciclos naturales de inundación de la laguna, lo cual puede tener consecuencias futuras sobre el desarrollo de las comunidades del manglar.

Palabras clave: manglar, laguna costera, boca estuarina efímera, invertebrados intermareales, niveles de inundación, desecación, insolación, impacto antrópico.

\section{REFERENCIAS}

Acosta, V., Betancourt, R., \& Prieto, A. (2014). Estructura comunitaria de bivalvos y gasterópodos en raíces del mangle rojo Rhizophora mangle (Rhizophoraceae) en Isla Larga, Bahía de Mochima, Venezuela. Revista de Biología Tropical, 62, 551-565.

Bingham, B. L. (1992). Life histories in an epifaunal community: coupling of adult and larval processes. Ecology, 73, 2244-2259.

Cedeño, J., Jiménez, M., Pereda, L., \& Allen, T. (2010). Abundancia y riqueza de moluscos y crustáceos asociados a las raíces sumergidas del mangle rojo (Rhizophora mangle) en la laguna de Bocaripo, Sucre, Venezuela. Revista de Biología Tropical, 58 (Suppl. 3), 213-216.

Díaz, O., \& Liñero-Arana, I. (2006). Poliquetos (Annelida: Polychaeta) asociados a substratos artificiales en tres localidades del Golfo de Cariaco, Venezuela. Saber, $18,3-10$.

Díaz, M. C., \& Rützler, K. (2009). Biodiversity and abundance of sponges in Caribbean mangrove: indicators of environmental quality. Smithsonian Contributions to the Marine Sciences, 38, 151-172.

Elliott, T., Persad, G., \& Webber, M. (2012). Variation in the colonization of artificial substrates by mangrove root fouling species of the Port Royal mangrove lagoons in the eutrophic Kingston Harbour, Jamaica. Journal of Water Resource and Protection, 4, 377-387.

García, J. M., \& Palacio, J. (2008). Macroinvertebrados asociados a las raíces sumergidas del mangle rojo (Rhizophora mangle) en las bahías Turbo y El Uno, Golfo de Urabá (Caribe Colombiano). Gestión y Ambiente, 11, 55-66.

Hernández-Trejo, H., Priego-Santander, A., López-Portillo, J., \& Isunza-Vera, E. (2006). Los paisajes físico-geográficos de los manglares de la laguna de La Mancha, Veracruz, México. Interciencia, 31, 211-219.

Hogarth, P. J. (1999). The biology of mangroves. U.S.A.: Oxford University Press.

Hunting, E. R., Van der Geest, H. G., Krieg, A. J., Van Mierlo, M. B. L., \& Van Soest, R. W. M. (2010).
Mangrove-sponge associations: a possible role of tannins. Aquatic Ecology, 44, 679-684.

Lara-Domínguez, A. L., Day, J. W., Yáñez-Arancibia, A., \& Sáinz-Hernández, E. (2006). A dynamic characterization of water flux through a tropical ephemeral inlet, La Mancha Lagoon, Gulf of Mexico. In V. P. Singh \& Y. Jun Xu (Eds.), Coastal Hydrology and Processes: Proceedings of the AIH 25th Anniversary Meeting \& International Conference" Challenges in Coastal Hydrology and Water Quality" (pp. 413422). USA: Water Resources Publications, LLC.

Lenz, T. \& Boehs, G. (2011). Ciclo reproductivo del ostión de manglar Crassostrea rhizophorae (Bivalvia: Ostreidae) en la Bahía de Camamu, Bahía, Brasil. Revista de Biología Tropical, 59, 137-149.

Little, C., \& Kitching, J. A. (1996). The biology of rocky shores. USA: Oxford University Press.

Londoño-Mesa, M., Polanía J., \& Vélez, I. (2002). Polychaetes of the mangrove-fouling community at the Colombian Archipelago of San Andrés and Old Providence, Western Caribbean. Wetlands Ecology and Management, 10, 227-232.

Marques-Silva, N. S., Beasley, C. R., Paiva Gomes, C., Lima Gardunho, D. C., Tagliaro, C. H., Schories, D., \& Mehlig, U. (2006). Settlement dynamics of the encrusting epibenthic macrofauna in two creeks of the Caeté mangrove estuary (North Brazil). Wetlands Ecology and Management, 14, 67-78.

Márquez, B. \& Jiménez, M. (2002). Moluscos asociados a las raíces sumergidas del mangle rojo Rhizophora mangle, en el Golfo de Santa Fe, Estado Sucre, Venezuela. Revista de Biología Tropical, 50, 1101-1112.

Psuty, N. P., Martínez, M. L., López-Portillo, J., Silveira, T. M., García-Franco, J. G., \& Rodríguez, N. A. (2009). Interaction of alongshore sediment transport and habitat conditions at Laguna La Mancha, Veracruz, México. Journal of Coastal Conservation, 13, 77-87.

Quiceno, P. A. \& Palacio, J. A. (2008). Aporte al conocimiento de los macroinvertebrados asociados a las raíces del mangle (Rhizophora mangle) en la ciénaga $\mathrm{La}$ Boquilla, municipio de San Onofre, Sucre. Gestión y Ambiente, 11, 67-78.

Quirós, J. A., \& Arias, J. E. (2013). Taxocenosis de moluscos y crustáceos en raíces de Rhizophora mangle (Rhizophoraceae) en la Bahía de Cispatá, Córdoba, Colombia. Acta Biológica Colombiana, 18, 329-340.

Rajagopal, S., Van der Gaag, M., Van der Velde, G., \& Jenner, H. A. (2005). Upper temperature tolerances of exotic brackish-water mussel, Mytilopsis leucophaeata (Conrad): An experimental study. Marine Environmental Research, 60, 512-530.

Reyes, R., \& Campos C. N. H. (1992). Moluscos, anélidos y crustáceos asociados a las raíces de Rhizophora mangle 
Linnaeus, en la región de Santa Marta, caribe colombiano. Caldasia, 17, 133-148.

Romero-Murillo, P. E., \& Polanía, J. (2008). Sucesión temprana de la taxocenosis Mollusca-Annelida-Crustacea en raíces sumergidas del mangle rojo en San Andrés Isla, Caribe colombiano. Revista de Biología Marina y Oceanografia, 43, 63-74.

Ruiz, M. (2002). Diversidad de macroinvertebrados epibiontes de raices de mangle rojo (Rhizophora mangle L.) en la Laguna de La Mancha, Veracruz, México (Tesis de maestría). Instituto de Ecología, A.C., Xalapa, México.

Ruiz, M., \& López-Portillo, J. (2014). Variación espaciotemporal de la comunidad de macroinvertebrados epibiontes en las raíces de mangle rojo Rhizophora mangle (Rhizophoraceae) en la laguna costera de La Mancha, Veracruz, México. Revista de Biología Tropical, 62, 1309-1330.

Rützler, K. (1995). Low-tide exposure of sponges in a Caribbean mangrove community. Marine Ecology, 16, 165-179.

Schwindt, E., De Francesco, C. G., \& Iribarne, O. O. (2004). Individual and reef growth of the invasive reef-building polychaete Ficopomatus enigmaticus in a south-western Atlantic coastal lagoon. Journal of the Marine Biological Association of the United Kingdom, 84, 987-993.

Sokal, R. \& Rohlf, F. (1995). Biometry: the principles and practice of statistics in biological research. U.S.A.: W. H. Freeman.
Soto, M., \& García, E. (1989). Atlas climático del Estado de Veracruz. Publicación 25. Instituto de Ecología, Xalapa, Veracruz, México.

Sutherland, J. P. (1980). Dynamics of the epibenthic community on roots of the mangrove Rhizophora mangle, at Bahía de Buche, Venezuela. Marine Biology, $58,75-84$.

Tovar-Hernández, M. A., \& Yáñez-Rivera, B. (2012). Ficha técnica y análisis de riesgo de Ficopomatus miamiensis (Treadwell, 1934) (Polychaeta: Serpulidae). Capítulo X. In A. M. Low-Pfeng \& E. M. Peters Recagno (Eds.), Invertebrados marinos exóticos en el Pacífico mexicano (pp. 193-212). México: Geomare, A.C., INE-SEMARNAT.

Villalobos-Figueroa, A., De la Parra, R., Galván, B. E., Cacho, O. J., \& Izaguirre, M. A. (1984). Estudio hidrobiológico en la laguna de La Mancha, Municipio de Actopan, Veracruz. 1979-1980. Boletín del Instituto Nacional de Investigaciones sobre Recursos Bióticos, 15, 1-51.

Wulff, J. L. (2004). Sponges on mangrove roots, Twin Cays, Belize: early stages of community assembly. Atoll Research Bulletin, 519, 1-10.

Wulff, J. L. (2009). Sponge community dynamics on Caribbean mangrove roots: significance of species idiosyncrasies. Smithsonian Contributions to the Marine Sciences, 38, 501-514.

Zar, J. H. (1996). Biostatistical analysis. Third edition. New Jersey, USA: Prentice Hall. 
